\title{
Lattice Dynamics of CrW Dilute Alloy Using Modified Embedded Atom Method Potential
}

\author{
Manesh Chand $^{1 *} \cdot$ P D Semalty ${ }^{1}$ \\ ${ }^{1}$ Department of Physics, H. N. B. Garhwal University, S. R. T. Campus Badshahithaul, Tehri Garhwal- \\ 249199, India \\ *Corresponding Author Email: maneshchand@gmail.com
}

Received: 28.8.2021; Revised: 21.10.2021; Accepted:5.11.2021

(C)Society for Himalayan Action Research and Development

\begin{abstract}
A modified embedded atom method (MEAM) has been used to study the lattice dynamics and vibrational properties of $\mathrm{CrW}$ alloy. Using the MEAM potential the force-constants up to second neighbours for pure $\mathrm{Cr}$ and its dilute alloy with small concentration of $\mathrm{W}$ as substitutional impurity are calculated. The Phonon dispersions for dilute $\mathrm{CrW}$ alloy at $0.3 \%, 0.8 \%$ and $1.6 \%$ concentration of $\mathrm{W}$ substitutional impurity have been computed and the obtained results are compared with the available experimental data. We have obtained a very good agreement with the experimentally measured results of phonon dispersions. With the application of obtained force-constants from MEAM potential, the local vibrational density of states in ideal crystal and its alloys using Green's function method has been calculated. On the basis of the results of local vibrational density of states, the condition of resonance modes has been investigated. Using the calculated vibrational local density of states, the mean square thermal displacements of impurity atoms in $\mathrm{CrW}$ alloys are also calculated.
\end{abstract}

Keywords: Modified embedded atom method; Phonon dispersion; Green's function; force- constants; density of states; resonance modes; Mean square displacements.

PACS Nos: 61.72.S-; 61.72.Bb; 63.20. D-; 71.20.Be

\section{Introduction:}

In view of the inadequacy of embedded atom method (EAM) model of Johnson \& Oh (1989), an analytical modified embedded atom method (MEAM) was employed by Zhang et al $(1996,1999)$ in which an additional term depending on sum of square of electron density has been included to resolve the problem of the negative Cauchy pressure in Cr. This model has been widely applied to bulk systems and it has shown an ability to describe several physical properties of metals including of $\mathrm{Cr}$ and their alloys (Zhang et al op cit, Hu et al 2002a,b, Yuan et al 2003, Zhang et al 2003, Deng etal 2004, Zhang et al 2006).

$\mathrm{Hu}$ et al (2002a) have applied an analytic MEAM model proposed by Zhang et al (op cit) to calculate the phonon dispersions and some point defect properties of bcc transition metals. The vibrational properties of $\mathrm{CrW}$ alloys in which $\mathrm{W}$ is introduced as substitutional impurity with its very small concentration $0.3 \%, 0.8 \%$ and $1.6 \%$ was investigated experimentally to explore the possibility of resonance modes by Cunningham et al (1970) and found the expected resonant behaviour. The experimental results show a decrease in the frequencies after adding substitutional heavy impurity $\mathrm{W}$ compared with the phonon dispersions of pure Cr crystal. Theoretically, Garg et al (1984), and Imaizumi and Sukla (1993) have made the calculation of phonon dispersions and compared their results with the 
experimental results of Cunningham et al (op cit). It was found that the calculation of dispersion curves of $\mathrm{CrW}$ alloys obtained by Imaizumi and Sukla (op cit) show better agreement with the experiment compared to the results of Garg et al (op cit) In our present study, we have used the MEAM potential to compute the phonon dispersion curves at three different concentrations of $\mathrm{W}$ for $\mathrm{CrW}$ alloys in three symmetry directions (100), (110) and (111) and compared our results with the available experimental data (Cunningham 1970). The obtained phonon dispersion curves are found to be in good agreement with the experimental results. In our earlier studies of vibrational properties of vacancy in transition metals (Gairola et al 2013,2014), we have investigated the local density of states, vacancy formation entropy and mean square thermal displacements in bcc metals. As a result of substitution of the impurities in the pure metals, apart from the change in physical properties of metals, there is a possibility of excitation of characteristic modes. These characteristic modes, i.e., resonant or localised modes are observed as resonant peaks at lower frequencies or additional peaks at higher frequencies in the local density of states. In the presence of heavy impurity, as in the present case, or weak interactions between host and impurity atoms, there is a strong possibility of occurrence of resonant modes. The local density approach has been used to investigate the resonant vibrational behaviour in the metals (Dederichs and Zeller 1979 ). Rath and Ram (1989) have also presented the study of local density of states, resonant and local modes vibrations of substitutional impurities in $\mathrm{RbCl}$ and $\mathrm{KBr}$. As regards the local density of states of substitutional impurities, several studies have been made by Semalty and Kapil (2005), Semalty (2006) and Semalt et al (2006) in fcc metals by considering the pair potential term only. An investigation of vibrational properties of crystals with selfinterstitial point defects has also been made by Pohlong and Ram (2005) using EAM. In general EAM potential are quite successful in dealing with static properties of defects such as formation energy where large distortions around defect atom are there. However, these potentials are not so successful (Pohlong and Ram, 1998) in understanding the situations where small displacements are involved such as lattice vibrations. It is of some interest to use MEAM potential to study the lattice vibrations and properties of substitutional impurities. As the accuracy of the inter atomic potentials greatly affects the results related to various physical properties, in our present calculation we have followed the modified form of an EAM model employed by (Hu et al 2002a) in which the detail procedure for computing the MEAM potential parameters of pure bcc transition metals has been presented. In an attempt to gain deeper insight into the nature of point defects and to provide useful information for the interpretation of impurities in the transition metals, in the present work, in addition to the concentration dependent phonon dispersions of $\mathrm{CrW}$, we have also investigated the vibrational local density of 
states and mean square thermal displacements of $\mathrm{W}$ substitutional impurity atoms in the alloy using MEAM potential and compared the results with that of host metal $\mathrm{Cr}$.

\section{Theory:}

\section{MEAM Model:}

The total energy in the MEAM model (Zang et al op cit) can be expressed as:

$$
E=F(\rho)+\frac{1}{2} \sum_{m} \Phi\left(r_{m}\right)+M(P),
$$

where $F(\rho)=F\left(\rho_{\varepsilon}\right)\left[1-\gamma \ln \left(\frac{\rho}{\rho_{\varepsilon}}\right)\right]\left(\frac{\rho}{\rho_{\varepsilon}}\right)^{\gamma}$

is the embedding function and

$$
\phi(r)=k_{-1}\left(\frac{r}{r_{1}}\right)^{-1}+k_{0}+k_{1}\left(\frac{r}{r_{1}}\right)^{1}+k_{2}\left(\frac{r}{r_{1}}\right)^{2}+k_{3}\left(\frac{r}{r_{1}}\right)^{3}+k_{4}\left(\frac{r}{r_{1}}\right)^{4}
$$

(3)

is the pair-potential function [4].

The additional energy modification term is:

$$
M(P)=\sigma\left(1-\frac{P}{P_{\theta}}\right)^{2} \exp \left[-\left(\frac{P}{P_{\theta}}-1\right)^{2}\right]
$$

Force-constants can be obtained from the total energy as:

$$
\begin{aligned}
\Phi_{i j}(l, m)=\frac{\partial^{2} E}{\partial r_{i}^{l} \partial r_{j}^{m}}=- & \left\{\left[\phi^{\prime \prime}\left(r^{l m}\right)-\frac{\phi^{\prime}\left(r^{l m}\right)}{r^{l m}}\right] \frac{r_{i}^{l m} r_{j}^{l m}}{\left(r^{l m}\right)^{2}}+\delta_{i j} \frac{\phi^{\prime}\left(r^{l m}\right)}{r^{l m}}\right\} \\
& +\quad \sum_{n \neq l, m} F^{\prime \prime}\left(\rho_{n}\right) f^{\prime}\left(r^{l n}\right) f^{\prime}\left(r^{m n}\right) \frac{r_{i}^{l n}}{r^{i n}} r_{j}^{m n} \\
& +4 M^{\prime \prime}\left(P_{n}\right) \sum_{n \neq l, m} f^{\prime}\left(r^{l n}\right) f\left(r^{l n}\right) f^{\prime}\left(r^{m n}\right) f\left(r^{m n}\right) \frac{r_{i}^{l n}}{r^{l n}} \frac{r_{j}^{m n}}{r^{m n}}
\end{aligned}
$$

where $\quad$ are the atoms numbers and ${ }^{l, m} \quad$ are the Cartesian coordinates.

For alloys the force constants at different concentration can be obtained by the linear relation:

$$
\Phi_{i j}(\text { Alloy })=(1-x) \Phi_{i j}(A)+x \Phi_{i j}(B)
$$

where A, B are host $(\mathrm{Cr})$ and impurity (W) atoms and ${ }^{x}$ is the concentration of substitutional impurity. 
The phonon dispersions are calculated by diagonalizing the dynamical matrix, obtained from the Fourier transform of the forceconstant tensor ${ }^{\Phi_{\mathrm{ij}}}(\mathrm{l}, \mathrm{m})$.

\section{Local density of states:}

Local density of states has been discussed in detail by Dederichs et al (1979). The density of states of individual atom which is either host or impurity is called the local density of that particular atom. The total density can be expressed in terms of the sum of densities of states of all atoms present in the lattice. For the calculation of the local density of states of the substitutional impurities, we have used the Green's function method of Ram (1991). Local density of states can be expressed in terms of the imaginary part of Green's function of the defect lattice.

Assuming the presence of single impurity the Green's function of imperfect lattice in terms of ideal lattice Green's function, may be written as:

$$
G(\omega)=G^{0}(\omega)\left[I+V(\omega) G^{0}(\omega)\right\rceil^{-1}
$$

Where, $\left.V(\omega)=\left(\Phi-\Phi^{o}\right)-\left(M-M^{o}\right) \omega^{2}\right) \quad$ is the perturbation matrix due to single impurity.

The condition of the occurrence of resonance or localized mode may be given by:

$$
\operatorname{ReD}(\omega)=\operatorname{Re}\left|1+G^{0}(\omega) V(\omega)\right|=0,
$$

where $\operatorname{Re} D(\omega) \quad$ is the real part of the denominator $D(\omega)$.

In case of resonance we get an increase in the local density of states in lower frequency region. The local density of states of an atom 1 in pure lattice and in a lattice with substitutional impurity in direction can be expressed (Ram 1991) by the following expressions:

$$
Z_{\alpha}^{o}(l, \omega)=\frac{2 \omega M^{0}}{\pi} \operatorname{Im} G_{\alpha \alpha}^{o}(\omega)
$$

and

$$
Z_{\alpha}^{d}(l, \omega)=\frac{2 \omega M^{d}}{\pi} \operatorname{Im} G_{a \alpha}^{d}(\omega)
$$

where $\operatorname{Im} G_{\alpha \alpha}^{o}(\omega)$ and $\operatorname{Im} G_{\alpha \alpha}^{d}(\omega)$ are the imaginary parts of ideal and defect Green's function. 


\section{Mean Square thermal displacement:}

The mean-square thermal displacement of impurity and host atoms is another important property to know about the vibrational behaviour of alloys. The mean square thermal displacements can be expressed in terms of the local density of states of the atoms:

$<U_{\propto}^{2}>=\int \frac{z_{\alpha}(1, \omega)}{M \omega^{2}} \epsilon(\omega, \mathrm{T}) \mathrm{d} \omega$,

where $\epsilon(\omega, \mathrm{T})=\frac{\hbar \omega}{2} \operatorname{coth}\left(\frac{\hbar \omega}{2 \mathrm{~kg}_{\mathrm{B}} \mathrm{T}}\right)$, then

$<U_{\propto}^{2}>=\int \frac{\hbar z_{\propto}(\omega)}{2 M \omega} \operatorname{coth}\left[\frac{\hbar \omega}{2 k_{B} T}\right] d \omega$.

As shown in eq.(12), the mean square displacements is an integral of local density of states and in the presence of heavy substitutional impurity $\mathrm{W}$ atoms, mean square thermal displacements would reduce compared to that of host $\mathrm{Cr}$ atoms.

As for temperature dependence, at very low temperatures,

$\in(\omega, T) \rightarrow \frac{\hbar \omega}{2} \quad$ for $k_{B} T \ll \hbar \omega$

and at high temperatures

$$
\in(\omega, T) \rightarrow k_{B} T \text { for } k_{B} T \gg \hbar \omega
$$

We can see that the mean square displacement at high temperatures is especially large and increases linearly with temperatures due to increased thermal population of low-frequency modes.

\section{Results and discussions:}

The potential parameters and input data used in the calculation of MEAM potential are taken from (Zhang et al 1999). The model parameters $\sigma$ and k's (Eq. 3) were obtained analytically by fitting to the experimental elastic constants, cohesive energy, formation energy and lattice constant. With the help of these parameters the pair potential, embedded part and modified term are calculated taking only up to the second neighbour distances from impurities. Total energies of perfect crystal and crystal with substitutional impurity have been calculated using MEAM potential parameters and from the expression of total energy, corresponding force-constants are obtained. To compute the phonon dispersions for $\mathrm{CrW}$ alloys we have used force- constants obtained from equation (5). The phonon dispersion curves for $\mathrm{Cr} 0.3 \% \mathrm{~W}, \mathrm{Cr} 0.8 \% \mathrm{~W}$, and $\mathrm{Cr} 1.6 \% \mathrm{~W}$ (Figs. 1-3) are obtained for three symmetry directions (100), (110) and 
(111) and compared with the experimental results of (Cunningham 1970) available only

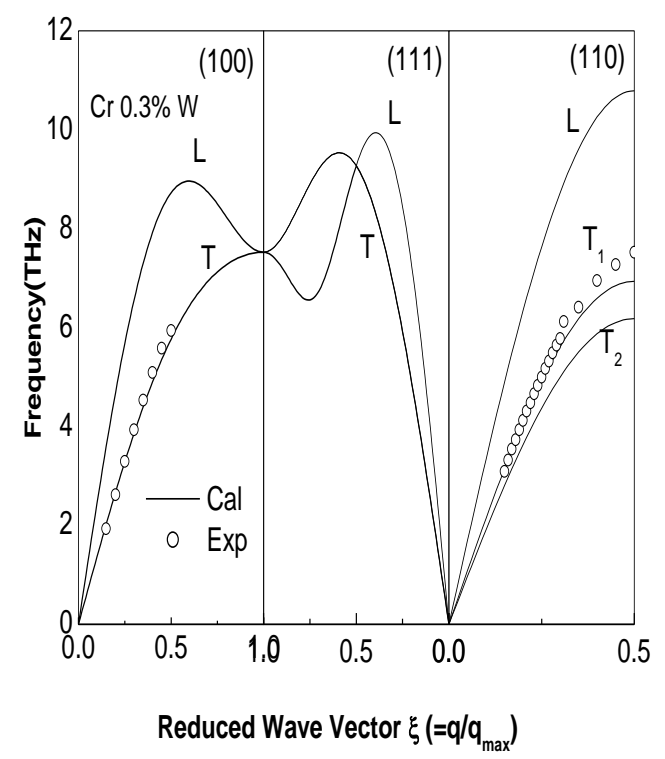

Fig. 1: Phonon dispersions for $\mathrm{Cr} 0.3 \% \mathrm{~W}$

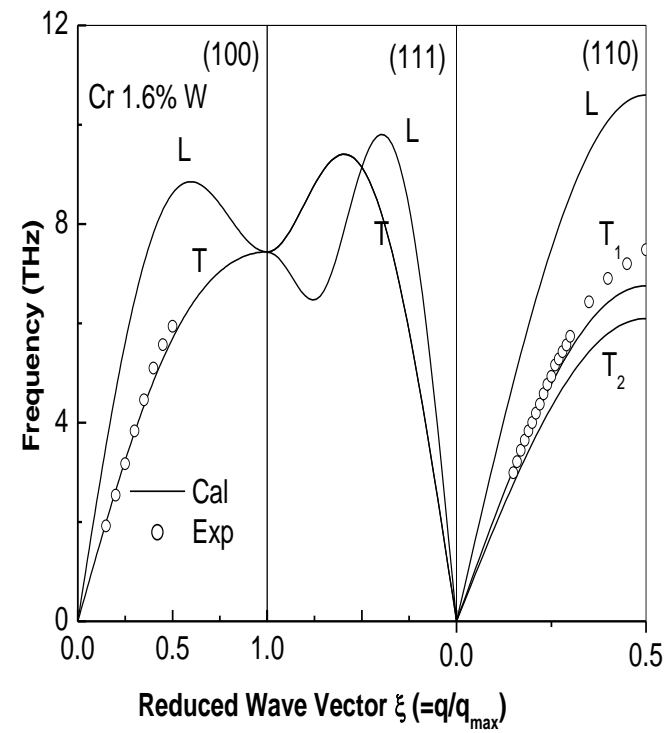

Fig. 3: Phonon dispersion for $\mathrm{Cr} 1.6 \% \mathrm{~W}$ in two symmetry directions (100) and (110).

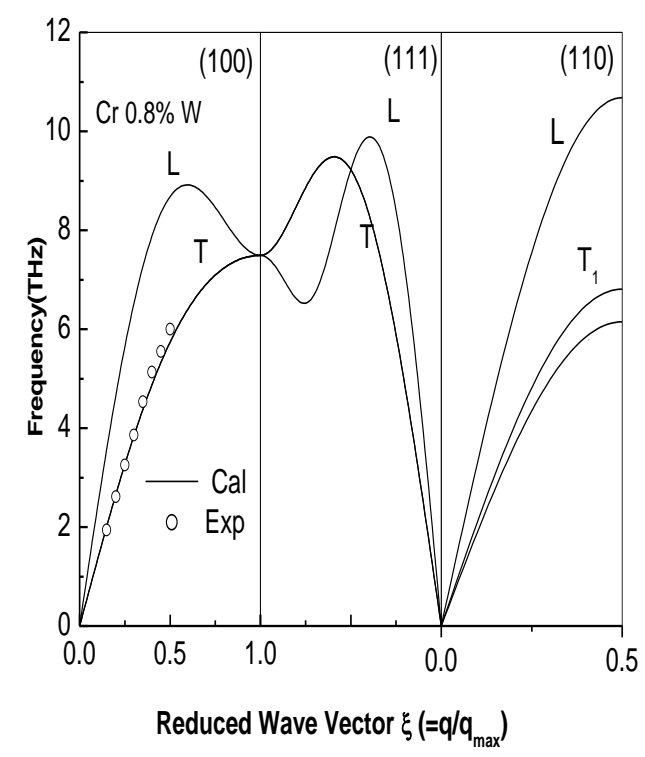

Fig. 2: Phonon dispersions for $\mathrm{Cr} 0.8 \% \mathrm{~W}$

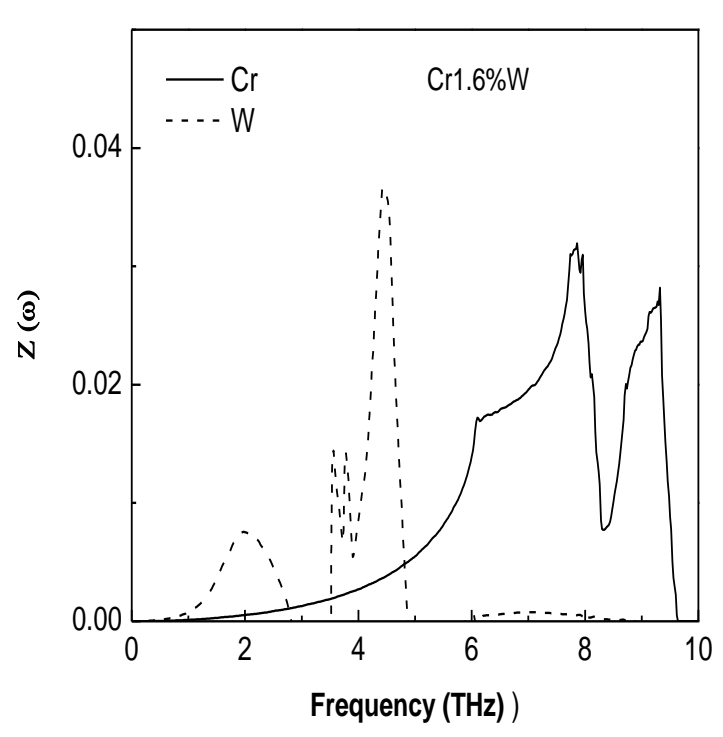

Fig. 4: Local density of states 
The calculated results show a very good agreement with the experimental results except near the zone boundary in (110) direction and obtained an overall improvement compared to the earlier calculations (Garg et al 1984, Imaizumi and Sulkla 1993 ). In order to further investigate the condition of resonant modes, we have calculated the local density of states using Green's function method. To compute ideal lattice Green's function, the required phonon data of $\mathrm{Cr}$ are obtained from (Shah and Muhlestein 1971 ) derived on the basis of the Born-Von-Karman fit to measured phonons in neutron scattering experiments. In the calculation of Green's functions, we have followed the modified Gilat-Raubenheimer method (Gilat and Raubenheime 1966). In the calculation of the frequency spectrum (local density of states) of pure $\mathrm{Cr}$ (solid curve) and impurity atoms (dotted curves) shown in Fig. (4), we have used the force-constants directly obtained from the MEAM potential using Eq. (5).

The calculated vibrational local density of states of host $\mathrm{Cr}$ shows the similar behaviours shown by this metal obtained on the basis of the Born-Von Karman fits to neutron scattering results. The local density of states of impurity atoms $\mathrm{W}$ shows an overall shift in the frequency spectrum towards lower frequencies and a prominent resonance peak is observed at $\omega=4.44 \mathrm{THz}$. This is an expected behaviour, keeping in view the heavy mass of impurity (more than three times) compared to that of host $\mathrm{Cr}$. To observe the condition of resonance the calculation of local density of states is presented only for $\mathrm{Cr} 1.6 \% \mathrm{~W}$ as for other concentrations i.e., $0.3 \%$ and $0.8 \%$, the results are almost similar due to very small changes in the force constants. As shown in Eq. (7), the defect Green's function, used in the calculation of local density of states, is directly affected by mass change and force-constant changes. Therefore, the increase in the local density of states at lower frequency in $\mathrm{CrW}$ alloy could be attributed to strong mass difference and weak coupling. This expected behaviour of vibrational local density of states of bec transition metal alloys is similar to that obtained for the fcc alloys (Semalty et al 2006).

The presence of resonant mode in the lower frequency region in $\mathrm{CrW}$ alloys investigated in the present study is in accordance with the general theory of vibrational properties of point defects (Elliott and Maradudin 1965, Maradudin et al 1971, Behra and Deo 1967). The mean square thermal displacements (Fig.5), vary linearly with temperature and a decrease in the mean square displacement of atoms in the alloy compared to the $\mathrm{Cr}$ atoms in the pure metal is observed. This behaviour of mean square displacement in the presence of heavy impurity has been reported earlier in the study of NbMo alloys by Powell et al (1977). The overall large decrease in the mean square displacement of $\mathrm{W}$ atoms is mainly due to the strong mass defect. 


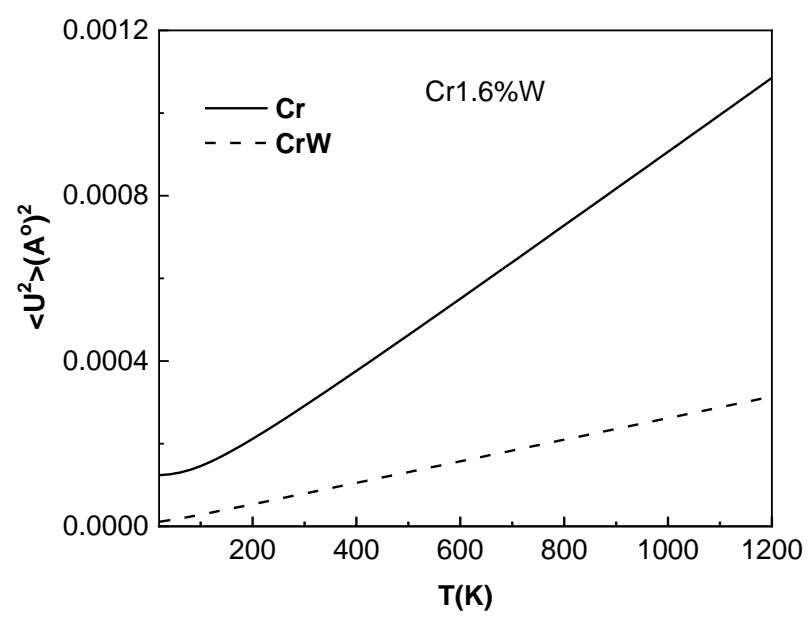

Fig. 4: Mean square thermal displacements

\section{Conclusions:}

We have carried out detailed theoretical calculation and investigated the lattice dynamics and vibrational properties of $\mathrm{CrW}$ dilute alloy. The force-constants for $\mathrm{CrW}$ dilute alloys are computed by using second-neighbour MEAM potential model. The obtained forceconstants are used to calculate the phonon dispersions at three different concentrations of W. Further, vibrational local density of states and mean square thermal displacements of atoms in pure $\mathrm{Cr}$ and $\mathrm{CrW}$ alloy have also been computed. As a result of alloying, the role of the mass defect and force-constants change in the vibrational properties of $\mathrm{CrW}$ has been investigated. The phonon dispersions obtained on the basis of MEAM potential shows a very good agreement with the experimental data. The observed resonance condition is consistent with the general theory of vibrational behaviour of point defects.

\section{References:}

Behra SN, Deo B (1967). Substitutional Defects and Resonant Modes in Solids. Phys. Rev. 153: 728

Cunningham RM, Muhlestein LD, Shaw WM, Thompson CW (1970). Investigation of In-Band Resonant Modes in Cr-W Alloys by Inelastic Neutron Scattering. Phys. Rev.B 2: 4864

Dederichs PH, Zeller R (1979). In Point Defects in Metals II vol. 87 Springer Tracts in Modern Physics (edited by G. Hohler and E. A. Niekisch) Springer, Berlin.

Deng H, Hu W, Shu X, Zhang B (2004). Analytic embedded-atom method approach to studying the surface segregation of $\mathrm{Al}-\mathrm{Mg}$ alloys. Applied surface Science 221: 408-414

Elliott RJ, Maradudin AA (1965). Inelastic Scattering of Neutrons (International Atomic Energy Agency, Vienna, Vol I: 231 
Gairola V, Semalty PD, Ram PN (2013). Vibrational properties of vacancy in bcc transition metals using embedded atom method potentials. Pramana 80: 1041-105

Gairola V, Semalty PD, Ram PN (2014). Vibrational properties of vacancy in bcc $\mathrm{Nb}$ using embedded atom method. Indian J. Phys. 88: 171-176

Garg Sadhana, Gupta HC, Bansal TK, Tripathi BB (1984). Phonon dispersion in dilute Cr-W alloys. Physica B 125: 293-296.

Gilat G, Raubenheime LJ (1966). Accurate Numerical Method for Calculating Frequency-Distribution Functions in Solids. Phys. Rev. 144: 390

$\mathrm{Hu}$ W, Masahiro F (2002). The application of the analytic embedded atom potentials to alkali metals. Mod. Simul. Mater. Sci. Eng. 10: 707726

Hu W, Shu X, Zhang B (2002). Point-defect properties in body-centered cubic transition metals with analytic EAM interatomic potentials. Comput. Mater. Sci. 23: 175-189

Imaizumi M, Sulkla MM (1993). Dispersion of Phonon Waves in a Binary Alloy of Chromium and Tungsten. Brazilian Journal of Phys. 23: 189-193

Johnson RA, Oh DJ (1989). Analytic embedded atom method model for bcc metals. J. Mater. Res. 4: 1195-1201

Maradudin AA, Montroll EW, Weiss GH, Ipatova IP (1971). Theory of Lattice dynamics in the harmonic approximation, Solid State Physics Suppl.3 (Academic Press, New York: 353-514.

Ouyang Y, Chen H, Zhong X (2003). Enthalpies of Formation of Noble Metal Binary Alloys Bearing Rh or Ir. J. Mater. Sci. Technol. 19: 243-246

Ouyang Y, Zhang B, Shuzhi L, Jin Z (1996). A simple analytical EAM model for bcc metals including $\mathrm{Cr}$ and its application. Z. Phys. B 101: 161-168

Pohlong SS, Ram PN (1998). Analytic embedded atom method potentials for face-centered cubic metals. J. Metter. Res 13: 1919-1927

Pohlong SS, Ram PN (2005). Vibrational density of states of self interstinal atoms (SIas) in metals. Indian. J. Phys. 79: 973

Powell BM, Martel P, Woods ADB (1977). Phonon properties of niobium, molybdenum, and their alloys. Can. J. Phys. 55: 1601-1612.

Ram PN (1991). Vibrational properties of selfinterstitials in metals. Radiation, Effects and Defects in Solids 118: 193

Ram PN, Dederiches PH (1981). Resonant vibrations of self-interstitials in fccmetals. Z. Phys. B 42: 57-64.

Rath BK, Ram PN (1989). Gap Modes and Local Density of States of Impurities in $\mathrm{RbCl}$ and $\mathrm{KBr}$. Phys. Stat. Sol.(b) 156: $137-144$ 
Semalty PD (2006). Calculation of local density of states and lattice specific heat of gold- and silver-based dilute alloy at very low temperature. J. Alloys and compounds 419: 1-6

Semalty PD, Dev K, Ram PN (2006). Lattice specific heat and local density of states of Ni-based dilute alloys at low temperature. Pramana 66: 1077-1092

Semalty PD, Dev Kapil (2005). Lattice specific heat and local density of states of Pd0.95Ni0.05dilute alloys at low temperature. Indian J Phys. 79: 1279-1283

Shah WM, Muhlestein LD (1971). Investigation of the Phonon
Dispersion Relations of Chromium by Inelastic Neutron Scattering. Phys. Rev. B 4: 969

Yuan X, Takahashi K, Ouyang Y, Onzawa $T$ (2003). Development of a modified embedded atom method for bcc transition metals. J. Phys: Condens Matter 15: 8917-8926

Zhang B, Ouyang Y, Shuzhi L, Jin Z (1999). An analytic MEAM model for all BCC transition metals. Physica B 262: 218-225

Zhang JM, Wen YN, Xu KW (2006). Atomic simulation of the vacancies in $\mathrm{BCC}$ metals with MAEAM. CEJP 4: 481493 